\title{
The Change Research of Soil Moisture and Temperature in Farm Field

\author{
Yueling Zhao ${ }^{1, a^{*}}$,Junze $\mathrm{Li}^{1, \mathrm{~b}}$, Fanyu Liang ${ }^{1}$ and Weiqiang Ruan ${ }^{1}$ \\ ${ }^{1}$ Jilin Agricultural University, Changchun, 130118, China \\ azyueling@163.com, ${ }^{\mathrm{b}} 275714495 @ q q . c o m,{ }^{\mathrm{c}} 1358084016 @ q q . c o m$
}

\author{
Keywords: Soil temperature; Soil moisture; Time sequence; Model prediction
}

\begin{abstract}
Soil temperature and humidity is a dynamic part of the soil properties, it is an important factor of affect the soil fertility. Soil temperature and humidity in different periods of change trend is very different. During each period, according to the comparative analysis of forecast values and measure values simply from the perspective of a model fitting effect, short time interval effect is more ideal than long time interval, the relative error of them is both a hour of relative error is smaller than in days. By the curve estimation method for the forecast of temperature and humidity analysis ,we can see that temperature than the humidity is more suitable for using this method to forecast, the relative error is $1.74 \%$ and $7.60 \%$, respectively, relative errors are below $10 \%$. So in the later study, we can consider to use this method for the forecast of temperature.
\end{abstract}

\section{Introduction}

Soil temperature and humidity is a dynamic part of the soil properties, it is an important factor of affect the soil fertility, soil temperature and moisture distribution in time and space are often very uneven. Soil temperature directly affects crop sowing time, seed germination and sprout sooner or later, Mark Twain in a suitable temperature range are comparatively high, seed germination. On the other hand, temporal features of soil moisture is refers to some characteristics of soil moisture is dependent on time, such as soil water dynamic characteristics, change rule, etc., or refers to soil moisture changes over time and show the stability and variability, in order to emphasize the movement of soil moisture is dependent on time, to distinguish soil moisture depends on the change characteristics of spatial location. Soil moisture size determines the crops need to grow and supply and demand of water and soil purification ability itself. Low soil moisture means less soil water content, dry land. Such soil quality not only affects crop growth and production of late, more serious still can cause soil erosion rate, make the soil humus and plant organisms have not easily transformed and accumulation, which could lead to soil nutrient content is lower; If soil humidity is too high, and can lead to soil aeration, the plant roots respiratory function abate, at the same time also affect crop growth and fruit quality.

\section{Materials and Methods}

The research object of agricultural land is in jilin province, determination of the temperature of the tool with soil layers of temperature sensor, as shown in figure 4.1, the equipment is mainly collected at different depth of the soil temperature, and then uploaded to the controller, the acquisition of information is a digital signal, the information processing can be directly embedded into the processor, the tool can test 3-4 layer at the same time, the temperature of bus transmission, simple wiring, measuring precision, there was only collected $20 \mathrm{~cm}$ of soil temperature; Determination of soil moisture by intelligent soil moisture measuring instrument, the instrument by the type of SWR soil moisture sensor and measuring instrument and so on, as shown in figure 4.2 is the measured data on site at the time, it can pass the LCD directly read data; Can also be via a standard serial interface connected to the computer, and can make the measured data in the form of text stored in the computer. 
The Concept of Time Sequence. A time series is a series of observations made in time order .First used in the $1880 \mathrm{~s}$ western economists and statisticians of capitalist economic cycle and business forecasting research. It shows that the development of social economic phenomenon change process and its development trend, say again so dynamic sequence. In all kinds of social economic phenomenon in the time series is to be the important basis of dynamic analysis and forecast. It is a kind of historical data analogy and extension of predictions, it is primarily through research and analysis of time series data, according to the time sequence of development direction, development process to predict the next period of time or after a longer period of time is likely to reach the level of development and target .

In record time series, the general purpose according to the different requirements and choose different record unit, the commonly used method, season, month, day, and grading unit, also because of different unit of time so the data is divided into low frequency data, high frequency, ultra high frequency data. Usually put in a year frequency of relatively less data, referred to as the low frequency data; Week of high frequency data refers to record the data for the unit, such as; And ultra-high frequency data to a within minutes or hours of recorded data for the unit, discusses its own rule for different types of data, we usually use methods and models should also is different.

The Characteristics of Time Series and Function. In time series analysis, although variable related to time, but it is not necessarily the strict time $t$ function, it has a certain value at a certain moment in randomness, overall, time series usually present a trend or a cyclical changes. Time sequence specific role is as follows:(1) the time-series model is the extension of dynamic model with dynamic data features, most of the literature indicates that using the time series analysis than, cycle with the method of correlation analysis method of statistical properties obtained by the methods such as accurate.(2) all levels index and speed index, the study on the development of the social and economic phenomenon.(3) in the different time sequence into the same model is analyzed, the direction of the phenomena in the tip, and as a result, the speed and dynamic comparison, to achieve the objective of the laws of understanding and mastering.

Stationary Time Series Model Identification and Estimation. (1) three kinds of model identification of the model. Analysis of a stationary time series, the first on the pattern recognition, this is the first step on the fit model. That determine the sequence of the model appropriate for the type. Here need to use time series autocorrelation function (ACF) and partial autocorrelation function (PACF) auxiliary judgment [168], for a balance of time series, we can calculate the stationary time series samples of sample autocorrelation function and partial autocorrelation function, and then compared with the characteristics of one kind of model, thus the model appropriate for the preliminary judgment sequence types. In order to further determine the stand or fall of model, the commonly used criterion function of order, namely to determine a criterion function, when modeling according to the value of the criterion function size to determine the merits of the model (2) the model selection method. Information criterion method selection plays a very important role in the model, the questions about its fixed order, generally choose the following two criteria :The first is the AIC Criterion method: AIC Criterion English full name is the Akaike's Information Criterion of red pool Information Criterion, was established in 1973 by Akaike proposed, considering both the Criterion of data fitting model proximity, is considered in the model contains the number of parameters. About the ARMA (p, q), AIC function to its definition are as follows:AIC $(p, q)=n \ln \left(\sigma^{\Lambda 2}\right)+2(p+q)$. The second is BIC rule method: Akaike AIC criterion was improved in 1976, BIC criterion is put forward. Thus avoided in large sample cases, AIC criterion in choosing order convergence is bad faults. About the ARMA (p, q), BIC function to its definition are as follows: $\operatorname{AIC}(p, q)=n \ln \left(\sigma^{\Lambda 2}\right)+2(p+q) \ln n$. White noise sequence is a special kind of stationary series, is the essential characteristic that distinguishes it from other sequence: Covariance of the variables of this sequence in the difference to 0 , all the useful information in the time series have been extracted, the rest is random interference information, and cannot be used to predict and, 
from the historical data information to speculate about the future of data information, also can terminate the modeling process, the sequence is also referred to as thel"no sequence of memory".

\section{Results and Analysis}

Soil Temperature and Humidity of the Trend Analysis of Time Series. Through statistical analysis of the experimental area of the experimental data of soil temperature and humidity, analysis and make the time sequence diagram of soil temperature and humidity, which is intuitive found their changing trends and characteristics, by means of correlation analysis, the temperature and humidity do know their correlation coefficient is 0.949 , the changes of soil temperature and soil moisture in time with a similar change trend.

Hours Prediction. This study selected a small time interval of temperature and humidity data, by using DPS software, curve estimation, through a moving average, exponential smoothing, linear regression model, the secondary moving average, the secondary exponential smoothing, a smoothing model of exponential smoothing analysis, three times, respectively, to get their decision coefficient, residual error, relative error and other information. By comparing three exponential smoothing curve model of analog effect is relatively good, decision coefficient is 0.94 , the relative error is only $1.74 \%$, the second times smoothing prediction decision coefficient is 0.98 , the relative error is only $2.75 \%$, the relative error of the linear regression model is $4.1 \%$, and residual error is 0.85 , it is relative to the fitting effect is not good. So if we use the curve estimation method to predict the temperature of high frequency data, we should choose the three exponential smoothing model.

According to fitting the minimum mean square error, observation system shows a variety of trend analysis, choose a relatively good model, it is selected three exponential smoothing model, and to determine the related parameters of the model as shown in figure 4.6, such as smoothness when $=$ 0.9 , and the mean square error is 0.4068 , three exponential smoothing is a kind of time series prediction method, the prediction model is: $Y(t+1)=a(t)+X(1-a) Y(t)$. In the formula, $X t+T$ means the year of the base year, the prediction period for the predicted period of $\mathrm{T}$; $\mathrm{T}$ is the prediction period, $\mathrm{a}, \mathrm{b}$, and $\mathrm{c}$ are all smoothing coefficients. $\mathrm{Y}(\mathrm{t}+\mathrm{t})=17.6997-0.2914 \mathrm{t}+0.0161$ T2.According to the fitting of the minimum mean square error, the contrast analysis of all kinds of system as a result, choose a relatively good model, it selected is an exponential smoothing model, the relative error is $20.83 \%$, and determine the related parameters of the model such as the smoothing parameter when $=0.98$, here is minimum mean square error (16.6810). Therefore, the system determines the prediction model based on $=0.98$. The forecast results in $X(t+1)=99.8806$ (the soil humidity at 5:59 GMT on September 12, 2008).

Days prediction. This is experimental data a day, measuring time is sep 201206 solstice December 11, 2012 11:59 measured value of time, every day after analysis and processing, all the parameters are shown in table 4 to 5 below: in the day as the unit of temperature time series prediction model can be seen in the comparative analysis, the relative error less than $10 \%$ of exponential smoothing, once the secondary exponential smoothing, a smooth model, three exponential smoothing model, four smallest an exponential smoothing, its relative error is $7.60 \%$, determination coefficient of 0.85 . In the units of day moisture time series prediction model can be seen from the analysis, the relative error less than $10 \%$, can be seen from the results of the study applied curve estimation method to forecast the humidity, effect. In the curve prediction, the relative error of the model can only be compared to 223 percent, and the decision coefficient is 0.72 .

Comparison Analysis. The temperature and humidity of the soil are very different in different time periods. During each period, according to the comparative analysis of forecast values and measure values simply from the perspective of a model fitting effect, short time interval effect is more ideal than long time interval, the relative error of them is both a hour of relative error is smaller than in days. But the curve estimation method for the forecast of temperature and humidity 
analysis we can see that temperature than the humidity is more suitable for using this method to forecast, take hours or days as the unit of temperature prediction relative error is $1.74 \%$ and $7.60 \%$, respectively, relative errors are below 10\%. Temperature measured every 3 hours of numerical prediction with three exponential smoothing model and decision coefficient reached 0.94 , so in the later study can consider to use this method for the forecast of temperature and humidity can apply this method to predict remains to be further studied and confirmed its feasibility.

\section{Discussion}

The change in soil moisture is a continuous process in time, and the change in humidity of a soil over a certain period of time produces a sequence of time. Many researchers have long been interested in studying soil temperature and humidity prediction models. In the study of the relationship between soil temperature and temperature, zhu found that the temperature of the soil fluctuates according to the fluctuation of temperature, and the trend of change is basically consistent . The study found similar trends in soil temperature and soil moisture. Due to a high reliability model to model the required parameters, the limitation of such factors as the complexity of model itself, make it in the actual extension and prediction precision problems more or less, etc, and has published some research results to some extent, the lack of practicability and multi-scale universality, so through the analysis of long-term monitoring data of soil moisture, soil moisture time series prediction, for soil moisture forecast methods provide theoretical basis for the improvement and to increase the prediction precision.

\section{Acknowledgments}

Funds for the research was provided by scientific research fund of Jilin Agricultural University(No.201620),Jilin province university undergraduate science and technology innovation fund-portable soil comprehensive indicator tester; spark plan Ministry of science and technology (2015GA660004).

\section{References}

[1] ZhengXiaohuan. local government to promote energy conservation and emissions reduction countermeasures research [D]. 2014 tianjin university of commerce.( In Chinese).

[2] Xu Junyan. Discussion on the application of regression analysis and forecasting method in the financial budget of the [J]. financial sector, the 5 phase of the 2011 146-147

[3] Zhu Dongmei, Huang Daichun, Hu Jianhua, Wang Qingxin, application of [J]. ARIMA time series model in budget revenues of telecom products in micro computer information, 2006, 22 (6-3): 178-17930

[4] Wang Lijie, Liu Zhidong economic time series analysis technology in coal price forecasting [J]. Journal of China coal society, 2001, 26 (1): 109-112

[5] Ya-bin xu. research and practice of University-enterprise cooperation personnel training mode [J]. computer education .2013 (2): 1-4. (In Chinese).

[6] Zhang Ya, Xiao Dongrong, Chen Keyan, Wang Dong. Prediction model of time series of Shenzhen's [J]. statistical analysis and decision, in 2008 533-34

[7] C. Narendra Babu, B. Eswara Reddy .A moving-average filter based hybrid ARIMA-ANN model for forecasting time series data[J]. Original Research Article Applied Soft Computing, 2014, 23(10):27-38

[8] Yun-linzhao. local undergraduate course of teaching reform breakthrough [J]. practice teaching:2008 (12): 51-52. (In Chinese). 
[9] Zhi-guo sun, Hai-ping xu. the teaching reform discussion situation of energy saving and emission reduction in environmental systems [J]. Journal of education teaching exploration (9): 2012-60. (In Chines

[10] Ertur C,Koch W,Regional disparities in the European Union and the enlargement process: An exploratory spatial data analysis, 1995-2000. Annals of Regional science, 2006, 40(4): 723-765 